\title{
COMPONENT PROPERTIES OF SECOND ORDER LINEAR SYSTEMS
}

BY SHAIR AHMAD AND A. C. LAZER

Communicated by François Treves, October 16, 1975

Consider the second order linear system

$$
x^{\prime \prime}+A(t) x=0,
$$

where $A(t)$ is an $n$-by- $n$ continuous matrix. Disconjugacy and other Sturm-type properties of solutions of (1) have been studied by a number of people (see e.g. [1]-[4]). Virtually no study has been made of the sign properties of the individual components of solutions of (1). It appears that such a study would be of interest not only from a theoretical point of view but also from a more practical point of view. We announce some results along this line, which are obtained under certain conditions on the matrix $A(t)$. For definitions and basic concepts one might consult [1] and [4].

THEOREM 1. Let $A(t)=\left(a_{i j}(t)\right)$ be symmetric with $a_{i j}(t) \geqslant 0$ whenever $i \neq$ $j$ and $t \in[a, b]$, where $b$ is the first conjugate point of $a$. Then there exists $a$ nontrivial solution $u(t)=\operatorname{col}\left(u_{1}, \ldots, u_{n}\right)$ of $(1)$ with $u(a)=u(b)=0$, and $u_{k}(t) \geqslant 0$ on $[a, b], k=1, \ldots, n$.

The converse of Theorem 1 holds in the following sense.

THEOREM 2. Assume that $A(t)=\left(a_{i j}(t)\right)$ is positive definite on $(a, b)$ except at isolated points. If $a_{i j}(t)>0$ on $(a, b)$, and if there exists a nontrivial solution $y(t)=\operatorname{col}\left(y_{1}, \ldots, y_{n}\right)$ of $(1)$ with $y(a)=y(b)=0$ and $y_{i}(t) \geqslant 0, i=$ $1, \ldots, n$, on $(a, b)$, then $b$ is the first conjugate point of $a$.

We recall that an $n$-by- $n$ matrix $A=\left(a_{i j}\right)$ is called irreducible if it is impossible to have $\{1,2, \ldots, n\}=I \cup J, I \cap J=\varnothing, I \neq \varnothing \neq J$, and $a_{i j}=0$ for all $i \in I, j \in J$.

THEOREM 3. Let $A(t)=\left(a_{i j}(t)\right)$ such that $\left.a_{i j}(t)\right) \geqslant 0$ on $[a, b]$ and $A\left(t_{0}\right)$ is irreducible for some $t_{0} \in(a, b)$. If $y(t)=\operatorname{col}\left(y_{1}, \ldots, y_{n}\right)$ is a nontrivial solution of (1) such that $y(a)=y(b)=0$ and $y_{i}(t) \geqslant 0$ on $(a, b), i=1, \ldots, n$, then $y_{i}^{\prime}(a)>0, y_{i}^{\prime}(b)<0$ and $y_{i}(t)>0$ on $(a, b), i=1, \ldots, n$. Moreover, if $w(t)$ is any solution of (1) with $w(a)=w(b)=0$, then $w(t)=\alpha y(t)$ for some constant $\alpha$.

AMS (MOS) subject classifications (1970). Primary 34C10. 
Theorem 4. Assume that $A(t)=\left(a_{i j}(t)\right)$ is symmetric with $a_{i j}(t) \geqslant 0$ on an interval $[c, \infty)$, and $A\left(t_{0}\right)$ is irreducible for some $t_{0}>c$. If $(1)$ is disconjugate on $[c, \infty)$, then there is a solution $y(t)=\operatorname{col}\left(y_{1}, \ldots, y_{n}\right)$ of $(1)$ satisfying $y(c)$ $=0$ and $y_{i}(t)>0$ on $(c, \infty), i=.1, \ldots, n$.

Let $P[a, b]$ denote the set of absolutely continuous $R^{n}$-valued functions $h(t)$ on $[a, b]$ such that $\left|h^{\prime}\right| \in L^{2}[a, b]$, and $h(a)=h(b)=0$. Let $J[h]$ define the functional

$$
J[h]=\int_{a}^{b}\left(\left\langle h^{\prime}, h^{\prime}\right\rangle-\langle A(t) h, h\rangle\right) d t .
$$

over the set of admissible functions $P[a, b]$. The following lemma is a slight modification of a well-known result (see [4, p. 332]).

LEMma 1. If $A(t)$ in (1) is symmetric and if $[a, b]$ contains no point conjugate to $a$ in its interior, then $J[h] \geqslant 0$ for all $h \in P[a, b]$.

To give an idea concerning the proof of Theorem 1 , we note that by definition of conjugate point there is a nontrivial solution $y(t)$ of $(1)$ with $y(a)=y(b)$ $=0$. It follows that $J[y]=0$. Let $u(t)=\operatorname{col}\left(u_{1}, \ldots, u_{n}\right)$, where $u_{i}(t)=$ $\left|y_{i}(t)\right|, i=1, \ldots, n$. Clearly, $u \in P[a, b]$. One then shows that $J[u] \leqslant J[y]$. This, along with Lemma 1 , implies that $J[u]=0$. It then follows from standard argument in the Calculus of Variations that $u(t)$ is the required solution (note that $u$ affords a minimum to $J$ for the class $P[a, b])$.

Next, we give an indication of the proof of Theorem 3. The first part of this theorem follows essentially from the definition of irreducibility and the following lemma.

LemmA 2. Let $A(t)=\left(a_{i j}(t)\right)$ with $a_{i j}(t) \geqslant 0$, and let $y(t)=\operatorname{col}\left(y_{1}, \ldots, y_{n}\right)$ be a solution of (1) satisfying $y(a)=y(b)=0$, and $y_{i}(t) \geqslant 0$ on $(a, b), i=$ $1, \ldots, n$. If for some $k, k=1, \ldots, n$, either (i) $y_{k}^{\prime}(a)=0$, (ii) $y_{k}^{\prime}(b)=0$, or (iii) $y_{k}(c)=0$ for some $c, a<c<b$; then $y_{k}(t) \equiv 0$ on $[a, b]$.

To prove the second assertion of Theorem 3, one shows that the set $\{\alpha>$ $0 \mid y_{i}(t)-\alpha w_{i}(t)>0$ on $\left.(a, b), i=1, \ldots, n\right\}$ is nonempty and bounded. Let $z^{*}$ $=y(t)-\alpha^{*} w(t)$, where $\alpha^{*}$ is the least upper bound of the preceding set. If one assumes that $w$ is independent of $y$, then it follows from the first part of the theo rem that $z_{i}^{*}(t)>0$ on $(a, b), i=1, \ldots, n$. One then shows the existence of a sufficiently small $\beta>0$ such that $z_{i}^{*}(t)-\beta w_{i}(t)=y_{i}(t)-\left(\alpha^{*}+\beta\right) w_{i}(t)>0$ on $(a, b), i=1, \ldots, n$, thus contradicting the definition of $\alpha^{*}$.

Proofs of Theorems 2 and 4 are based on a consideration of conjugate points relative to certain perturbations of (1), and applications of the above lemmas and Theorems 1 and 3 .

It can be shown that with the aid of certain transformations some of our theorems can be generalized considerably in the sense of weakening the requirements 
on the signs of the elements $a_{i j}(t)$ of $A(t)$. For example, Theorem 1 may be stated as follows:

Theorem 1'. Assume that $A(t)=\left(a_{i j}(t)\right)$ in (1) is symmetric, and let $b$ be the first conjugate point to a. Suppose that $\{1,2, \ldots, n\}=P \cup N$ with $P \cap$ $N=\varnothing$ and

(i) $a_{i j}(t) \geqslant 0$ on $[a, b]$ if $i \neq j$ and either $i, j \in P$ or $i, j \in N$,

(ii) $a_{i j}(t) \leqslant 0$ on $[a, b]$ if $i \neq j$ and either $i \in P, j \in N$ or $i \in N, j \in P$.

Then there exists a nontrivial solution $y(t)=\operatorname{col}\left(y_{1}, \ldots, y_{n}\right)$ of (1) such that $y(a)=y(b)=0, y_{k}(t) \geqslant 0$ on $[a, b]$ for $k \in P$, and $y_{k}(t) \leqslant 0$ on $[a, b]$ for $k \in N$.

\section{REFERENCES}

1. W. A. Coppel, Disconjugacy, Lecture Notes in Math., vol. 220, Springer-Verlag, Berlin, 1971.

2. I. M. Gel'fand and S. V. Fomin, Calculus of variations, Fizmatgiz, Moscow, 1961; English transl., Prentice-Hall, Englewood Cliffs, N. J., 1963. MR 28 \#3352; \#3353.

3. M. Morse, The calculus of variations in the large, Amer. Math. Soc. Colloq. Publ., vol. 18, Amer. Math. Soc., Providence, R. I., 1934.

4. W. T. Reid, Ordinary differential equations, Wiley, New York, 1971. MR 42 \#7963.

DEPARTMENT OF MATHEMATICS, OKLAHOMA STATE UNIVERSITY, STILLWATER, OKLAHOMA 74074

DEPARTMENT OF MATHEMATICS, UNIVERSITY OF CINCINNATI, CINCINNATI, 